\title{
Loading Silica for Supporting Carbon Black Filler to Prevent Degradation Natural Rubber Caused DME
}

\author{
Asep Handaya SAPUTRA ${ }^{1 *}$, Tuti Indah SARI ${ }^{2}$, Dadi R. MASPANGER ${ }^{3}$, and Setijo BISMO ${ }^{1}$ \\ ${ }^{1}$ Chemical Engineering Department FT-UI, UI New Campius, Depok, 16424, Indonesia \\ ${ }^{2}$ Chemical Engineering Department FT-Unsri, Kampus Indralaya, Indonesia \\ ${ }^{3}$ Indonesian Rubber Research Institute, Jl. Salak 16151 Bogor, Indonesia
}

\begin{abstract}
DME (Dimethyl Ether) is the future environmental friendly fuel. Some parts of equipment for transporting the DME are using rubber as a hoses or seals. This research is about the using of natural rubber for those applications. The influential part in the manufacture of natural rubber products are fillers. The use of filler depends on what the product will be made. DME nature have high permeability and easy to absorb into a rubber and plastic. In such of that, the rubber or plastic could be damaged and not durable. In order to determine the type of degradation, the number of samples were immersed in liquid DME. The ratio of the loading of carbon black/silica filler in natural rubber was varied. DME causes two types of degradation, namely absorption and extraction. The addition of filler composition can reduce the absorption and extraction, which can cause a reduction in the percentage decrease in the value of the change in mass and a decrease in tensile strength. On the other hand, the addition of filler composition will increase the changes of hardness. The higher filler loading, will increase the crosslink density and lower scorch time. The presence of silica further is to enhance the crosslink density as well as to lower scorch time. Therefore, the presence of silica affect on the decreasing of the swelling level and shrinking. In general, the presence of silica filler in the mixture will be slightly lowering the tensile strength, but not affecting the elongation at break. The presence of silica before and after soaking with DME will increase hardness.
\end{abstract}

\section{Introduction}

The products from limited natural rubber has major drawbacks that cannot stand the oil/solvent is an ester group, ether, hydrocarbons (some in the form of gas). Natural rubber will be degraded when in contact with $\mathrm{oil} / \mathrm{solvent}$ is an ester group, ether and hydrocarbons. If it is compared with synthetic rubber, natural rubber has special properties, i.e. elasticity, tensile strength, tear strength and high adhesion.

The causes of changes in the mechanical properties natural rubber in oil/solvent among others caused by the permeation of the liquid into the natural rubber that can cause swelling. Swelling may occur because of the permeability of rubber caused due to exposure to liquids rubber solubility or diffusivity of small molecule liquid into the rubber (Wood-Adams, 2006). Swelling caused dissolution occurs because of the similarity in polarity, such as the theory of "like dissolve like" (Ebewele, 2000), while the swelling caused by fluid diffusivity of small molecules that caught the network (network) in the rubber (Wood-Adams, 2006). Swelling may cause a decrease in properties such as hardness, tensile strength, tear strength and others.
Fluid permeation into the rubber also can cause shrinkage volume or mass. This is caused by attacked by the fluids in the chemical structure of the composition in the vulcanized rubber. It affects decreasing of the rubber physical properties.

Changes in the physical properties of natural rubber is affected by the materials constituent vulcanized rubber in natural rubber vulcanization process. One of the constituents of vulcanized rubber that is highly influential in the mechanical properties of natural rubber that is filler. Filler is also known as reinforcement, which means the amplifier is to strengthen the vulcanized natural rubber. It can also act as a filler in the network crosslinks on the rubber to cover the space in the rubber macromolecules capable of reducing diffusivity of liquids or gases get into the network.

On the other side each liquid or gas has different properties such as different ester groups with ether or a hydrocarbon group. Diffusivity of liquid or gas respectively to show the strength of natural rubber to absorb in the network on natural rubber.

Several previous studies that discuss the events absorption of oil/solvent in natural rubber and filler vulkanisator influence has done. As performed by Mathew et al, studied the oil absorption of toluene and n-

\footnotetext{
* Corresponding author: sasep@che.ui.ac.id
} 
hexane in natural rubber with filler Isora fiber (Mathew, et al., 2006). Absorption of toluene in natural rubber with filler oil palm ash (OPA) was studied by Ooi, et al., 2013a, with variations of 0-9 phr (part per hundred rubber) followed by a comparison with the filler silica and carbon black (Ooi, et al., 2013b). Research conducted by Ahmed et al. (2014), studied the absorption of toluene in the vulcanized rubber with silica (from rice husk) and marble mud as filler.

The use of DME as a substitute for LPG in Indonesia, as revealed in the discussion group Energy Resource Development Technology Center (PTPSE) Agency for the Assessment and Application of Technology (BPPT) (Ikawati, 2012). Previous study focuses only on the distribution system DME with synthetic rubber. Now in this research will study the durability of natural rubber vulcanized to the DME and changes in mechanical properties by testing the fluid absorption DME with effect filler. Based on the variation of the number and type of filler are carbon black and silica, will be studied its effect on the absorption and the percentage change in the mechanical properties of the vulcanized natural rubber.

\section{Experiment}

\subsection{Materials}

The raw material of natural rubber used was Standard Indonesia Rubber 10 (SIR10). All of the compound components consist of natural rubber (SIR10), vulcanisator (sulphur), activator (stearic acid), activator (zinc oxide), accelerator (2mercaptobenzothiazole/MBT), filler (carbon black N330) and plasticizer (minarex-B). All of the components were mixed in the open roll mill Berstorff with a rotor speed 20 and $28 \mathrm{rpm}$. After that, rubber compound from each sample was vulcanized in press molding at temperature $150^{\circ} \mathrm{C}$. The formulation of the vulcanized natural rubber are tabulated in Table 1.

Table 1. Formulation used in the preparation of the natural rubber vulcanized

\begin{tabular}{lc}
\hline \multicolumn{1}{c}{ Ingredients } & Formulation $\left(\right.$ phr $\left.^{*}\right)$ \\
\hline Natural rubber (SIR10) & 100 \\
Zinc Oxide (ZnO) & 5 \\
Stearic acid & 2 \\
Carbon black/Silica & $30 / 0 ; 20 / 10 ; 10 / 20 ; 0 / 30 ;$ \\
& $50 / 0 ; 40 / 10 ; 30 / 20 ; 20 / 30 ;$ \\
Minarex-B & $10 / 40 ; 0 / 50$ \\
2-mercaptobenzothiazole & 10 \\
(MBT) & 1.8 \\
Sulfur & 2.4 \\
$*$ phr $=$ part per hundred rubber &
\end{tabular}

The samples of the vulcanized rubber were soaked in dimethyl ether liquid phase in the pressurized vessel, 1.3 $\mathrm{L}$ of volume. The mass change of these samples were investigated after the samples had been soaked in DME for 7 days and then exposed to the atmosphere for 24 hours (Wu et al., 2008). The mass change is specified in percentage unit (\%) as shown in the equation (1) ( $\mathrm{Li}$ and Zhou, 2008; Wu et al., 2008; Trakarnpruk and Porntangjitlikit, 2008; Abu-Abdeen and Elamer, 2010).

$$
\text { The mass change }(\%)=\frac{m_{f}-m_{i}}{m_{i}} \times 100 \%
$$

where $m_{\mathrm{f}}$ (final mass) is the mass of the sample after being soaked and $\mathrm{m}_{\mathrm{i}}$ (initial mass) is the mass of the sample before being soaked. After measuring the mass of change of the samples, the tensile strength, elongation at break as well as hardness were tested.

\section{Results And Discussion}

\subsection{Effect of composition of filler carbon black/silica on cure characteristics}

Figure 1 shows that a cure characteristic data based on the influence of vulcanized natural rubber filler with the ratio carbon black/silica. Characteristics based on optimum cure, the difference between the maximum and minimum torque will explain the effect of loading on the filler carbon black/silica. Silica can affect the characteristics cure of vulcanized natural rubber.

Optimum cure time ( 190$)$, is the time required for the torque to reach $90 \%$ of the maximum achievable torque (T90). (Khimi and Pickering, 2014; Hariwongsanupab et al., 2017). Figure 1 shows that increasing silica loading, the optimum cure decreases. Compared with natural rubber without filler, natural rubber by using a filler mix with different ratios, the optimum cure average lower. Optimum cure shows inversely proportional tendency to the differences of torque. Torque $e_{\text {max-min }}$ value increasing with increasing filler loading. Exceptions occurred in the composition of the carbon black/silica 50/0 experiencing

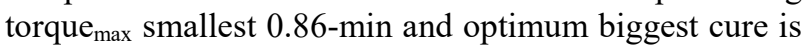
$9.95 \mathrm{~min}$.

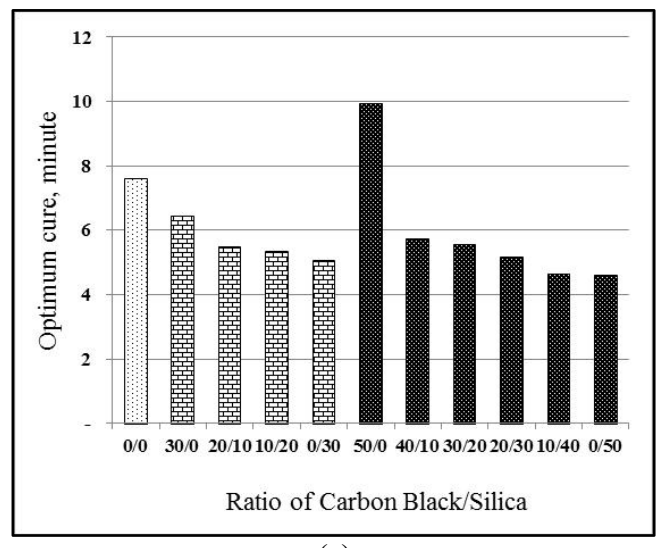

(a)

2.2 Mechanical property measurement 


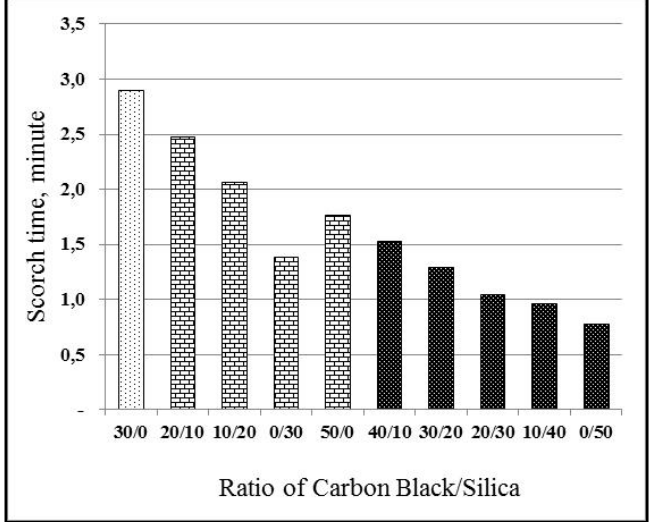

(b)

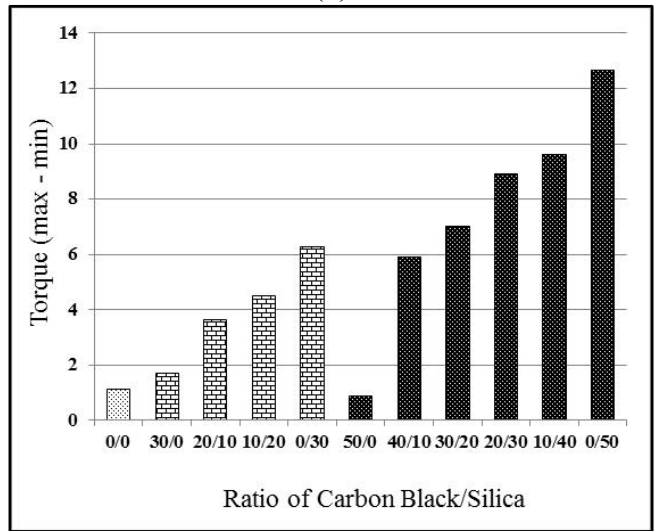

(c)

Figure 1. Optimum cure (a), scorch time (b) and torquemax-min (c) based on the ratio of carbon black/silica

Figure 1 shows the scorch time decreasing with increasing filler loading. The more silica also lowers the scorch time. According to research conducted Rahiman, it can be said the decline optimum cure and scorch time caused by the increase of acrylonitrile in NBR. From this condition can be stated that the presence of other compounds can acrylonitrile affect the optimum cure and scorch time (Rahiman et al., 2005).

\subsection{Effect of Composition Filler Carbon Black/Silica to Compression Set}

Compression set is defined as the permanent deformation that takes place is lower than the yield point of the material. It is similar to creep, cold flow, or stress relaxation. Sometimes the elastomer material fails to return to its original size after release of the compressive load is constant. The amount of material that failed to return to its original size is called compression set. Type of failure is the same for both static and dynamic seals. Failure compression set to produce a flat surface on both sides of the cross section of the O-ring, which reduces the potential for sealing. Some of the causes of failure in compression set, the choice of elastomer with a set of properties of low compression, materials with low thermal resistance, the degree of swelling high material to the fluid surrounding it, the level of compression to achieve high seal, the vulcanization process is not perfect in the production of o-ring.
Data compression set in Figure 2 below shows that the value of the natural rubber compression set is affected by loading and type of filler. The higher filler loading, then the value will be even greater compression set. Likewise, the type of filler effects on this phenomena. The presence of silica will increase the value of compression set.

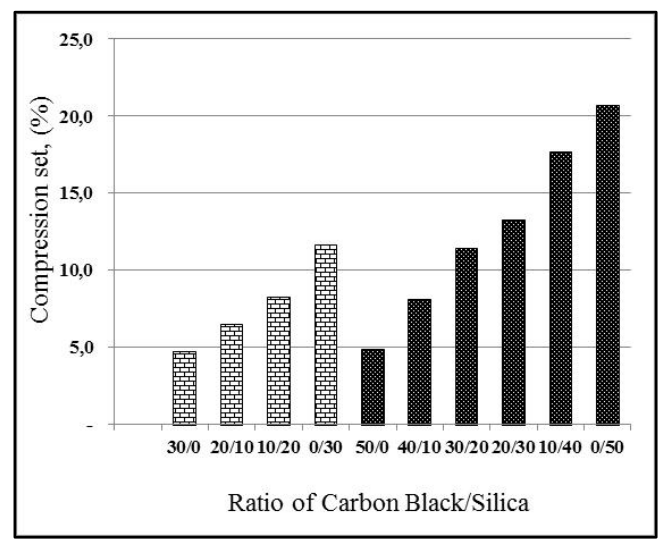

(a)

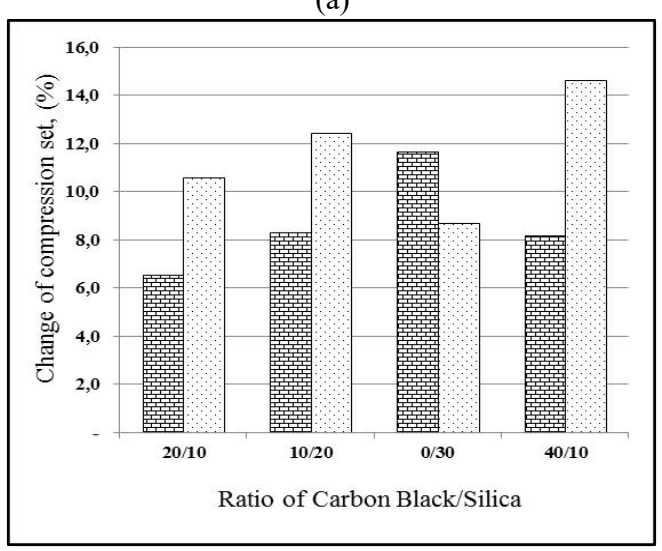

(b)

Figure 2. Compression set based on the ratio of Carbon Black/Silica (a) Compression set after soaking with DME (b)

\section{3 \\ Effect of composition filler carbon black/silica to swelling}

Data on the swelling for each sample was obtained through the calculation of the data on the mass before and after immersion in dimethyl ether (DME). Samples were made in three pieces shaped dumbbell. The aim is to improve the accuracy of the acquisition value of the masses, especially the mass after immersion. Values that are only used as discussion material will only be a middle value (median), either for the value of mass before and after immersion in DME as a marinade.

Based on this mechanism, the order of occurrence of these two types of degradation for this experiment can be explained as follows. Dimethyl ether is a compound of polar tendencies gathered on the cluster electrons of oxygen atoms. Therefore, these compounds are certain to interact with one or more chemicals in vulcanized natural rubber which is also a polar compound.

Data percent mass changes can be reviewed based on variations in the composition of the filler carbon 
black/silica contained in the sample. Filler loading will reduce the increase in the mass and lose mass after immersion. The same thing happens to the sample after they had been soaked in DME for 7 days and then exposed to the atmosphere for 24 hours. The influence of the filler on the mass loss of samples to be reduced.

Mass measurements of samples twice in the span of time apart meant is to investigate the existence of two types of degradation, adsorbed and extracted in each sample. This is necessary in view of absorption of the DME to natural rubber vulcanized is not necessarily preceded by the extraction of natural rubber vulcanized and its components by the DME. The basis is the percentage change in the value of the mass, where a positive value indicates the absorption and negative values indicate extraction. If the direct measurement of the mass was found that the percentage change is positive and then the assay after 24 hours of drying turns into a mass change is negative, it can be stated that in this sample absorption occurs at a time of extraction. On the other hand, a sample can be declared only degraded form of absorption when change of the mass is positive on direct measurements and remain positive (or zero) after 24 hours.

As shown in Figure 3, all samples confirmed with an absorption and extraction for DME marinade ingredients. Both types of degradation may arise because of a mechanism called "like dissolves like" (Haseeb et al., 2011; Li and Zhou, 2008). This mechanism basically stated that a chemical can dissolve other chemicals as long as it has a degree of the same polarity. That is, the only polar compounds can dissolve polar compounds and non-polar compounds also only against non-polar compounds alone. It is tailored to the type of intermolecular forces most dominant of each type of these compounds. Intermolecular forces, dominant in the polar compounds are dipole-dipole interactions, while the London dispersion forces for non-polar compounds (Haseeb et al., 2011).

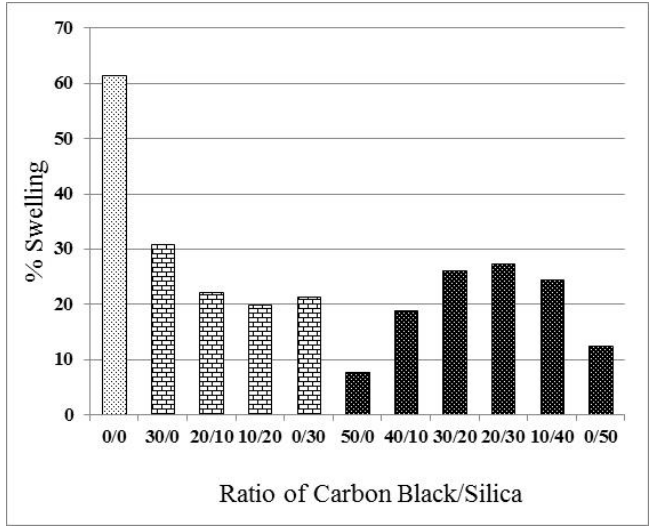

Figure 3. Percentage of swelling for samples immersed in DME

Figure 3 shows that the composition of the filler carbon black and silica each providing a negative influence on absorption. The greater the filler loading levels of both carbon black and silica to make the percentage of swelling decreases. At $30 \mathrm{phr}$ filler loading, use of carbon black filler provides a higher degree of swelling compared to using silica. However, at $50 \mathrm{phr}$ filler loading, carbon black provides a lower degree of swelling than with silica. Carbon black relatively nonpolar compounds, so it is not able to interact with DME. Therefore, molecules of carbon black can provide protection against the interaction of molecules of dimethyl ether. On the other hand, carbon black will also cover cavity in polymer structures that form a network in the vulcanization process, so that DME cannot move in. On the other hand, the silica filler without carbon black also has a small swelling. Mix the filler carbon black/silica results are not too good in the percentage of swelling. Chances are this is due to the incompatibility of carbon black and silica, resulting in the mixing process between the two fillers does not blend well. This causes the cavities so that the liquid DME easily fit into the rubber and cause swelling percentage increase. The lower the percentage of swelling is associated with torque $_{\max \text {-min }}$ values are higher with the addition of the silica filler. As Kapgate statement, that the swelling associated with crosslink density. The decrease swelling showed a high crosslink density in the rubber. According

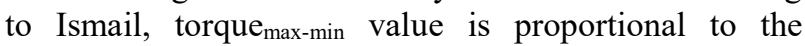
crosslink density in the rubber (Kapgate et al., 2012); (Ismail and Suzaimah, 2000).

However, it is not so visible in Figure 4, the filler carbon black and silica actually increases the percentage of shrinking after the sample dries. Compared with no filler, the filler carbon black and silica increases the mass loss occurs due to absorption and a good mix of carbon black/silica or carbon black and silica separately.

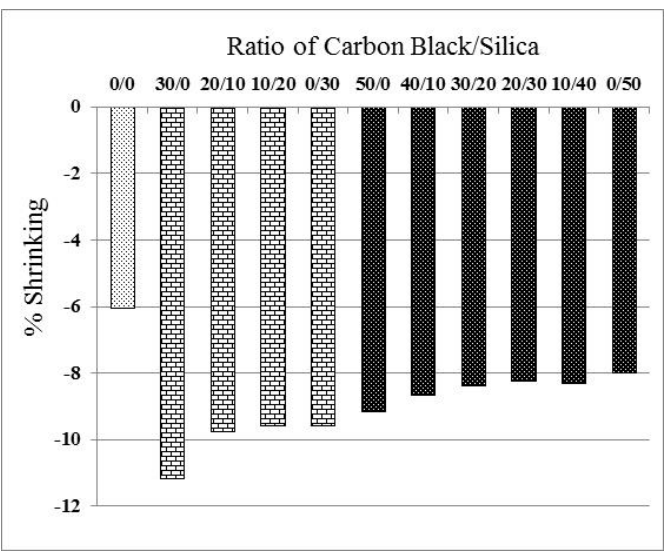

Figure 4. Percentage of shrinking after immersion in DME

\subsection{Effect of Composition Filler Carbon black/Silica on Tensile Strength and Tensile Strength Change due Soaking in DME}

Just as the percentage change in mass, tensile strength change data is not obtained directly from the calculation. The surface area of the sample and the maximum tensile strength reached before the sample is dropped out of the two types of data that should be sought in determining the tensile strength changes. In the group discussion is only the disclosed data on changes in tensile strength for each sample, of course, already a middle value (median). 


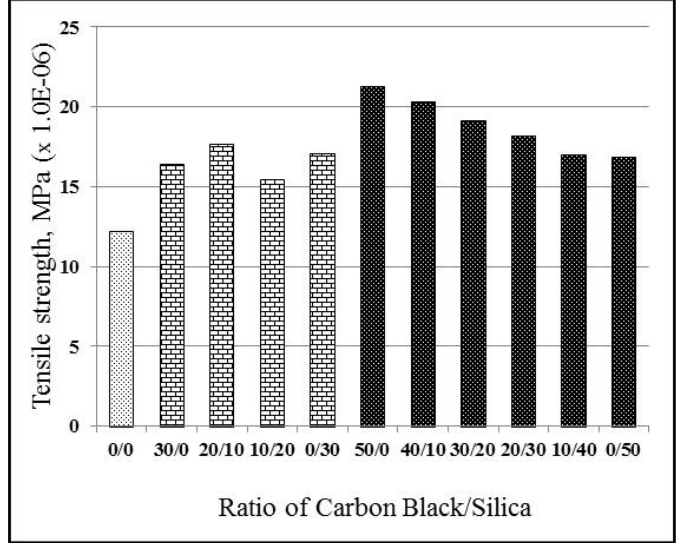

(a)

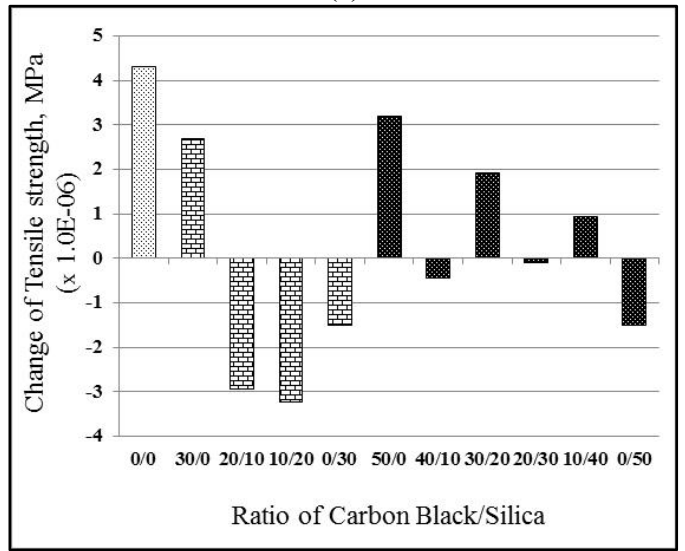

(b)

Figure 5. Tensile Strength (a) and changes after immersion in DME (b)

Based on the graph in Figure 5 (a) and 5 (b), in general, it can be stated that soaking the samples in DME provides value of tensile strength changes were negative, in other words a decrease in tensile strength. This occurs because the extraction of plasticizer, cause the formation of cavities in the polymer structure vulcanized natural rubber. The material is shredded as the solvent has the potential to infiltrate into the cavity. This absorption was also thought to weaken the bonds between the polymer and it ingredients, as by the pressure of the solvent from the outside (Haseeb et al., 2011). Weak ties have certainly weakened the strength of the vulcanized natural rubber in resisting all external forces, including gravity. Thus, the tensile strength of the final sample is smaller than the first.

The involvement of the variation of composition filler carbon black/silica associated also with changes in tensile strength obtained. Figure 5(a) and 5(b), state that the decline in negative changes in tensile strength occurs by reason of immersion will be smaller due to the addition of the silica filler mixture. The explanation for these events is the protection of surface and vulcanized natural rubber structure given by each of the molecules of carbon black. Carbon black also act as a protector of the extraction and degradation, because it does not interact with DME. The greater the amount of carbon black that exist, the better the protection. Therefore, a decrease in tensile strength can be reduced.

\subsection{Effect of Composition Variation Filler Carbon black/Silica to Hardness Changes after Immersion}

Hardness is known inversely proportional to the tensile strength. Both the graphs, Figure 6(a) and 6(b), showing that immersion in dimethyl ether will increase the hardness vulcanized natural rubber. This increase can occur as a result of coating by carbon black on the surface or cavity of vulcanized natural rubber. The presence of silica makes the material a little softer so easily extracted by the DME. Carbon black is a material that gives hardness to the material, so it came to pass that the violence will increase after immersion. From Figure 6()a and 6(b), it is known that generally the more positive change in hardness (increase in hardness after soaking) along with increasing the composition of the carbon black and plasticizer. The addition of carbon black is a clear influence on the violence.

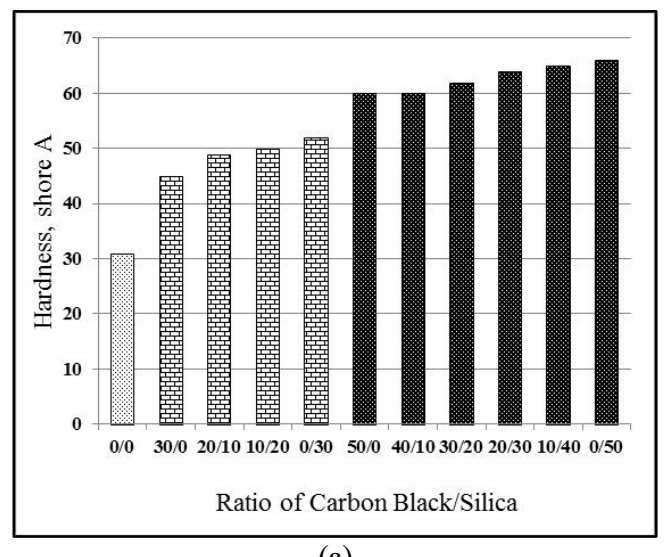

(a)

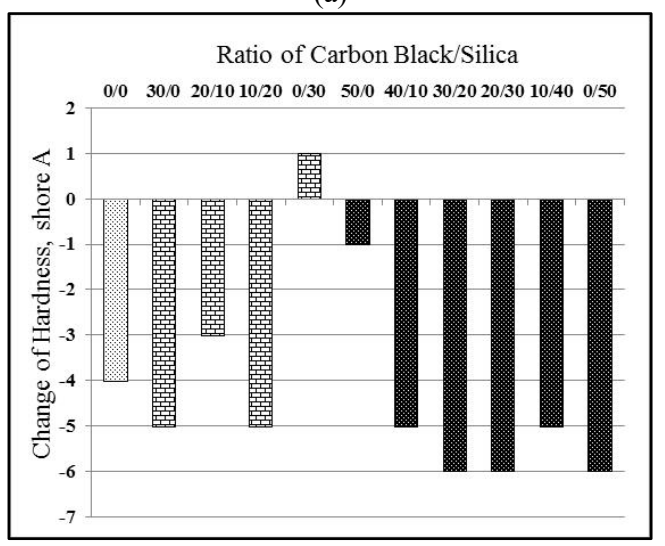

(b)

Figure 6. The hardness (a) and changes after immersion in DME (b)

\section{Conclusions}

There are some points of the conclusions that can be obtained from these experiments, i.e.: (i) The addition of the composition of carbon black filler can reduce the absorption and extraction, which can cause a reduction in the percentage decrease in the value of the change in mass and a decrease in tensile strength and on the other increase the sample hardness changes; (ii) The higher 
filler loading, will increase the crosslink density and lower scorch time. The presence of silica further enhances the crosslink density and lower scorch time. So the presence of silica effect on decreasing the level of swelling and shrinking; (iii) In general, the presence of silica filler mixture slightly lower tensile strength. After soaking with DME, a sample with a mixture of silica increase the tensile strength. The presence of silica before and after soaking with DME will increase hardness.

\section{Acknowledgements}

The author would like to express gratitude to The Indonesian Rubber Research Institute, Intensification of Process Laboratory of Chemical Engineering Department, Faculty of Engineering Indonesia University.

\section{References}

Abu-Abdeen, M. and I. Elamer; "Mechanical and Swelling Properties of Thermoplastic Elastomer Blends," Materials \& Design, 31(2), 808-815 (2010)

Ahmed, K., S. S. Nizami and N. Z. Riza; "Reinforcement of Natural Rubber Hybrid Composites Based on Marble Sludge/Silica and Marble Sludge/Rice Husk Derived Silica," Journal of Advanced Research, 5(2), 165-173 (2014)

Ebewele, R. O.; Polymer Science And Technology, CRC Press, New York, U.S.A. (2000).

Hariwongsanupab, N., S. Thanawan, T. Amornsakchai, M-F. Vallat, and K. Mougin; "Improving The Mechanical Properties of Short Pineapple Leaf Fiber Reinforced Natural Rubber by Blending with Acrylonitrile Butadiene Rubber," Polymer Testing, 57, 94-100 (2017)

Haseeb, A. S. M. A., T. S. Jun, M. A. Fazal and H. H. Masjuki, "Degradation of Physical Properties of Different Elastomers upon Exposure to Palm Biodiesel," Energy, 36(3), 1814-1819 (2011)

Ikawati, Y., "Dimetil Eter Siap Gantikan Elpiji," Kompas, Jakarta, Indnesia (2012)

Ismail, H. and S. Suzaimah; "Styrene Butadiene Rubber/Epoxidized Natural Rubber Blends: Dynamic Properties, Curing Characteristics and Swelling Studies," Polymer Testing, 19(8), 879-888 (2000)

Kapgate, B. P., C. Das, A. Das, D. Basu, U. Reuter and G. Heinrich, "Effect of Sol-Gel Derived in situ Silica on the Morphology and Mechanical Behavior of Natural Rubber and Acrylonitrile Butadiene Rubber Blends," Journal of Sol-Gel Science and Technology, 63(3), 501509 (2012)
Khimi, S. R. and K. L. Pickering; "A New Method to Predict Optimum Cure Time of Rubber Compound Using Dynamic Mechanical Analysis," Journal of Applied Polymer Science, 131(6), https://doi.org/10.1002/app.40008 (2014)

Li, G. B. and L-B. Zhou ; "Experimental Research on the Resistance of Rubber Materials to Dimethyl Ether," Proceedings of the Institution of Mechanical Engineers, Part D: Journal of Automobile Engineering, 222(6), 975-978 (2008)

Mathew, L., K. U. Joseph and R. Joseph, "Swelling behaviour of Isora/Natural Rubber Composites in Oils Used in Automobiles," Bulletin of Materials Science, 29(1), 91-99 (2006)

Ooi, Z. X., H. Ismail and A. A. Bakar; "Synergistic Effect of oil Palm Ash Filled Natural Rubber Compound at Low Filler Loading," Polymer Testing, 32(1), 38-44, (2013a)

Ooi, Z. X., H. Ismail and A. A. Bakar, "Optimisation of Oil Palm Ash as Reinforcement in Natural Rubber Vulcanisation: A Comparison between Silica and Carbon Black Fillers," Polymer Testing, 32(4), 625-630 (2013b)

Rahiman, K. H., G. Unnikrishnan, A. Sujith and C. K. Radhakrishnan; "Cure Characteristics and Mechanical Properties of Styrene-Butadiene Rubber/Acrylonitrile Butadiene Rubber," Materials Letters, 59(6), 633-639 (2005)

Trakarnpruk, W. and S. Porntangjitlikit; "Palm Oil Biodiesel Synthesized with Potassium Loaded Calcined Hydrotalcite and Effect of Biodiesel Blend on Elastomer Properties," Renewable Energy, 33(7), 1558-1563 (2008)

Wood-Adams, P.; "Diffusion and Permeability in Polymers," Retrieved from http://users.encs.concordia.ca/ woodadam/GCH6101/Di ffusion $\% 20$ and $\% 20$ permeability $\% 20$ in $\% 20$ polymers.pdf (2006)

Wu, N., W. Zhang and Z. Huang, "Impact of Dimethyl Ether on Engine Seal Materials," Frontiers of Energy and Power Engineering in China, 2(3), 279-284 (2008) 